\title{
Intervention Strategies for the Poor Feeder in the Newborn Intensive Care Unit: External Pacing versus Imposed Regulation
}

Marjorie Meyer Palmer MA, NLP, CCC-SLP

NOMAS Licensed professional, Speech Pathologist

Founder/Director, NOMAS International. www.nomasinternational.org

$\mathrm{P}$ rior to the 1980's infants in the intensive care nursery who demonstrated feeding problems were described as "poor feeders" with a "weak" or "poor" suck. There was no distinction available at that time to further describe those infants who were unable to orally take sufficient calories to grow. Consequently, infants remained in the hospital for extended periods of time because of poor feeding. Then the 1980's saw the birth of the Newborn Individualized Developmental Care and Assessment Program (NIDCAP). ${ }^{1,2,3}$ Caregivers were suddenly made aware of the stress cues, signs, and signals that preterm infants demonstrated as they were struggling with feeding. Caregivers then began to provide infants with frequent "breaks" during feeding by removing the bottle from the mouth so that they could breathe, burp, or just rest after a very long sucking burst. ${ }^{4}$ This technique of providing "breaks" became known as External Pacing.

External Pacing has always been based upon the cues, signs, and signals given by the infant to the caregiver during feeding and is a consequence of the relationship between the infant and feeder. Since each caregiver has his/her own individual style and manner of positioning the infant; holding the bottle; and selecting the nipple, the infant may demonstrate different stress cues for each caregiver and the interpretation of these signs and signals is subjective on the part of the feeder. Providing "breaks" during a feeding using this technique of External Pacing has been most effective for older infants who are closer to term and who demonstrate longer sucking bursts such as with a continuous burst pattern of 10-30 sucks per burst with swallow and respiration occurring during the burst followed by only a brief pause. ${ }^{5}$

External Pacing was developed as a cue-based technique to aid infants who were experiencing discomfort or distress during feeding and, since the 1980's, has become very popular and is used effectively and frequently with both late preterm and sick term infants whose sucking bursts consist primarily of more than 10 sucks/burst. This intervention strategy has been effective because breathing appears to be the last function integrated into a successful feeding episode for the preterm infant. ${ }^{6}$

In the 1990's, with the development of the NOMAS (Neonatal Oral-Motor Assessment Scale), for the first time a distinction was made within the poor feeders; trained examiners were able to diagnose the suck pattern as either disorganized or dysfunctional. 7 A disorganized suck was defined as "a lack of rhythm of the total sucking activity" ${ }^{\prime}$ which refers to the incoordination of suck, swallow, and breathe. A dysfunctional suck was defined as "an interruption of the normal sucking activity by abnormal movements of the jaw and tongue".
Those infants who present with a disorganized suck are unable to self-regulate the suck/swallow/breath due to a lack of neurological maturation and respiratory support secondary to immaturity. Being able to coordinate the pharyngeal swallow with respiration is a difficult task for many young infants. It is possible, however, for the caregiver to regulate the suck/swallow/ breathe for these infants during their feeding using Imposed Regulation, a diagnostic-based intervention strategy that may be implemented following a diagnosis of a disorganized suck on the NOMAS. This does not refer to a specific technique but rather focuses on the goal for the infant and may be implemented differently for each infant.

Imposed Regulation is most effective with young infants who demonstrate too much variability in the number of sucks per burst or a transitional suck. ${ }^{10}$ It is difficult for many young infants to inhibit the sucking movement so as to be able to breathe which often results in oxygen desaturation or spells of deglutition apnea. ${ }^{11}$ Imposed Regulation is based on the definition and description of the normal immature sucking pattern that is demonstrated by preterm infants. This normal pattern is characterized by very short sucking bursts of 3-5 sucks per burst followed by a pause of equal duration during which the infant breathes and/or swallows. ${ }^{5}$ This pattern is a burst/pause pattern in which bursts and pauses are of equal duration which requires that the infant stop the sucking activity to pause and breathe. When an infant is unable to do this the caregiver may implement Imposed Regulation for the first minute of the feeding after which the infant may be able to self-regulate. Imposed Regulation of the suck/swallow/breathe requires that the caregiver stop the transfer of liquid after three nutritive sucks and swallows to allow the infant to pause and breathe. If the caregiver attempts to build in a pause after five sucks/swallows it will most likely not be successful. Some infants will already demonstrate deglutition apnea or oxygen desaturations after just three seconds of sucking and swallowing without breathing. Once the normal immature burst-pause pattern has been imposed for one minute the infant may feed well for the remainder of the feeding. If the infant continues to be unable to self-regulate the caregiver may provide Imposed Regulation for an additional minute always giving the infant an opportunity to self-regulate after each minute of Imposed Regulation.

The technique that is selected to implement Imposed Regulation is infant-dependent and should be carefully selected on an individual basis for each infant. Some of the techniques that have been successfully implemented include: finger feeding; use of alternative utensils; tipping the bottle nipple; removing 
the bottle nipple from the mouth; tipping the baby so as to empty the bottle nipple; and pinching the nipple closed to stop the flow, just to mention a few.

It is important to understand the differences in the purpose, goal, patient population, and implementation of these two intervention strategies that are used to improve oral feeding for infants in the intensive care nursery. An understanding of these two intervention strategies is necessary so that the infant's needs can be individually addressed by prescriptive intervention.

External Pacing:

1) Cue-Based

2) Used with older infants who demonstrate longer sucking bursts

3) Provides breaks when infant is stressed, tired, fussy, needs to breathe, etc.

4) Usually implemented by removing the bottle nipple from the mouth

5) Once the infant has recovered the bottle nipple is re-inserted

Regulation:

1) Diagnostic-Based

2) Used with younger infants who demonstrate too much variability in the number of sucks per burst or a transitional suck $\left(\mathrm{NOMAS}^{\oplus}\right)$

3) Provided for first minute of feeding; and re-introduced for one minute intervals as needed if infant is unable to selfregulate

4) Follows a diagnosis of disorganized suck $\left(\mathrm{NOMAS}^{\circledR}\right)$ and prevents stress and discomfort during feeding

5) Technique is individualized and infant specific

Imposed Regulation is also an effective intervention strategy for those infants who have problems during the esophageal phase of swallow. The esophageal phase of swallow in infants has received less attention over the years than the oral phase of swallow (sucking). Infants who have difficulty during the esophageal phase of swallow such as esophageal dysmotility; retrograde movement; or gastroesophageal reflux may have significant discomfort with feedings and are at greater risk of developing a sensory-based oral feeding aversion later. ${ }^{12}$ Since the average suck/swallow ratio is 1:1 per one second material may be unable to clear the esophagus at that rate. When esophageal nt during feeding and could result in aspiration ${ }^{13}$. Imposed Regulation will allow only three swallows followed by a pause of equal duration during which the material has time to clear the esophagus.

In addition, when Imposed Regulation is used for preterm infants during videofluoroscopic studies it will often serve to prevent aspiration that may occur secondary to inability to coordinate the pharyngeal swallow with respiration; fatigue; and esophageal dysmotility and/or retrograde movement.

External Pacing and Imposed Regulation are just two of the intervention strategies that may be used for infants who present with poor feeding. The $\mathrm{NOMAS}^{\bullet}$ also diagnoses those infants who have a dysfunctional suck for which very different intervention strategies and treatment techniques are recommended and are beyond the scope of this article that focuses on only two of the intervention strategies for infants who have difficulty with the coordination of suck/swallow/ and breathe.

References:

1. Als H, Lawhon g, Brown E, et.al., "Individualized behavioral and environmental care for the very low birth weight preterm infant at high risk for bronchopulmonary dysplasia: neonatal intensive care unit and developmental outcome". Pediatrics 1986,78:1123-1132.

2. Als H, Gilkerson L, Duffy F. et.al., "A three-center, randomized, controlled trial of individualized developmental care for very low birth weight preterm infants: medical, neurodevelopmental, parenting, and caregiving effects." Journal of Developmental and Behavioral Pediatrics 2003,24:399-408.

3. Als H, Lawhon g, Duffy FH, et.al., "Individualized developmental care for the very low-birth weight preterm infant medical and neurofunctional effects." Journal of the American Medical Association 1994, 272:853-858.

4. VandenBerg KA. "Behavioral issues for infants with BPD” In: Strategies for Total Patient Care. Neonatal Network. Petulama, CA, 1990,Chapter 6: 112-152.

5. Gryboski J. Gastrointestinal problems in the infant. In: Major Problems in Clinical Pediatrics Saunders: Philadelphia, PA 1975:17-47.

6. Vice FL. and Gewolb IH. "Respiratory patterns and strategies during feeding in preterm infants.” Developmental Medicine and Child Neurology 2008, 50(6):467-472.

7 Palmer MM, Crawley K, Blanco I. “The Neonatal Oral-Motor Assessment Scale: A Reliability Study”. Journal of Perinatology 1993,13(1): 28-35.

8 Crook CK., "The organization and control of infant sucking. Advances in Child Development and Behavior 1979,14:209-252.

9 Braun MA, Palmer MM. "A pilot study of oral-motor dysfunction in 'at-risk' infants." Physical and Occupational Therapy in Pediatrics. 1985/86, 5(4): 13-25.

10 Palmer MM. "Identification and management of the transitional suck pattern in premature infants." Journal of Perinatal and Neonatal Nursing, 1993,7(1): 66-75.

11 Hanlon MB, Tripp JH, Ellis RE, et. al., "Deglutition apnoea as indicator of maturation of suckle feeding in bottle-fed preterm infants." Developmental Medicine and Child Neurology $1997,39(8) ; 534-542$.

12 Jadcherla SR. "Gastroesophageal reflux in the neonate" Clinics in Perinatology. 2002 March, 29(1).

13 Wolf LS, and Glass RP. "Clinical feeding evaluation” In: Feeding and Swallowing Disorders in Infancy: Assessment and Management. Therapy Skill Builders, Tucson, AZ. 1992,85-147.

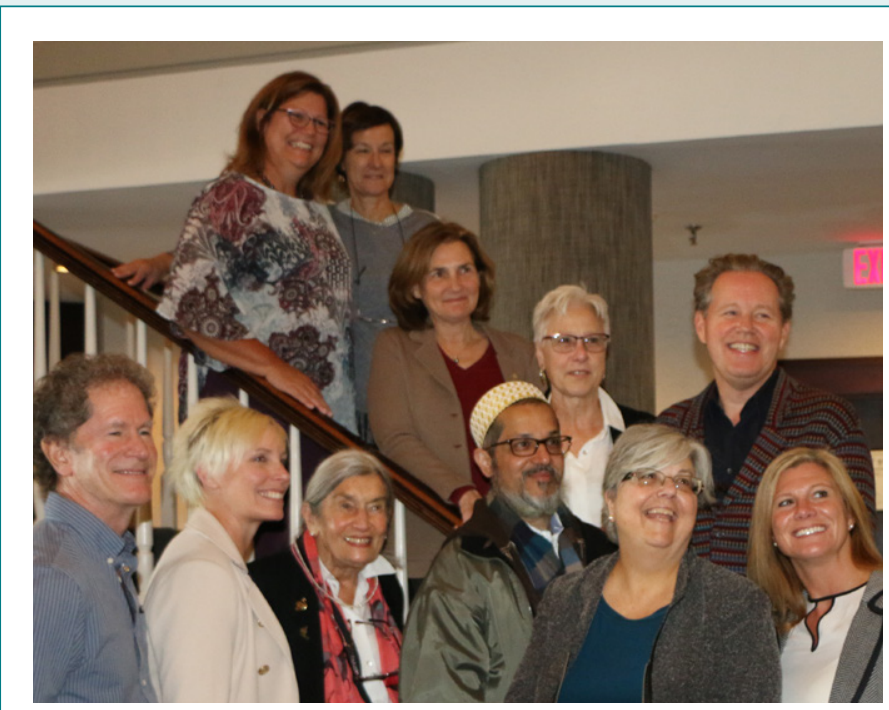

The $30^{\text {th }}$ Annual NIDCAP Trainers Meeting

Members of the 2018-2019 Board of Directors, Portsmouth, New Hampshire 